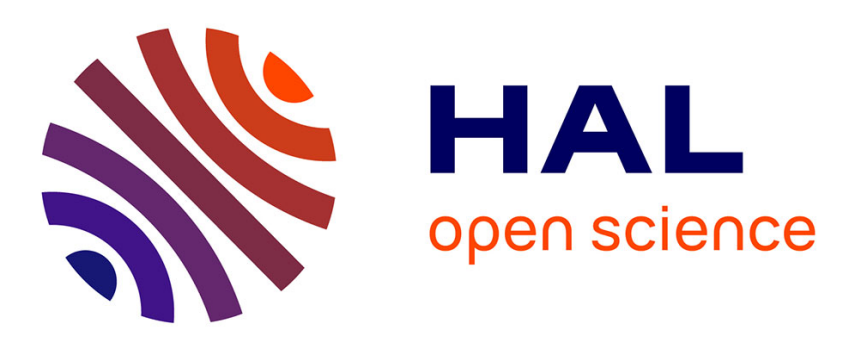

\title{
Identification of local contact angle distribution inside a porous medium from an inverse optimization procedure
}

\author{
Otman Maalal, Marc Prat, René Peinador, Didier Lasseux
}

\section{To cite this version:}

Otman Maalal, Marc Prat, René Peinador, Didier Lasseux. Identification of local contact angle distribution inside a porous medium from an inverse optimization procedure. Physical Review Fluids, 2021, 6 (10), pp.104307. 10.1103/physrevfluids.6.104307 . hal-03405047

\section{HAL Id: hal-03405047 https://hal.science/hal-03405047}

Submitted on 27 Oct 2021

HAL is a multi-disciplinary open access archive for the deposit and dissemination of scientific research documents, whether they are published or not. The documents may come from teaching and research institutions in France or abroad, or from public or private research centers.
L'archive ouverte pluridisciplinaire $\mathbf{H A L}$, est destinée au dépôt et à la diffusion de documents scientifiques de niveau recherche, publiés ou non, émanant des établissements d'enseignement et de recherche français ou étrangers, des laboratoires publics ou privés. 


\title{
Identification of local contact angle distribution inside a porous medium from an inverse optimization procedure
}

\author{
Otman Maalal, ${ }^{1,2}$ Marc Prat, ${ }^{1}$ René Peinador, ${ }^{2}$ and Didier Lasseux $\oplus^{3, *}$ \\ ${ }^{1}$ Institut de Mécanique des Fluides de Toulouse (IMFT), Université de Toulouse, \\ CNRS, 31400 Toulouse, France \\ ${ }^{2}$ Institut de la Filtration et des Techniques Séparatives (IFTS), \\ Rue Marcel Pagnol, 47510 Foulayronnes, France \\ ${ }^{3}$ I2M, UMR 5295, CNRS, Univ. Bordeaux - Esplanade des Arts et Métiers, 33405 Talence, CEDEX, France
}

\begin{abstract}
The local contact angle distribution within the pore space of a porous medium of mixed wettability is identified by combining pore network simulations and an optimization method. The latter is used to solve the inverse problem consisting of determining the local contact angle distribution from the capillary pressure and relative permeability curves. The inverse optimization method combines a genetic algorithm and the hill-climbing algorithm. The method is illustrated considering a Boolean distribution of the local contact angle in which the pore bodies and throats in the pore space are either hydrophilic or hydrophobic.
\end{abstract}

DOI: $10.1103 /$ PhysRevFluids.6.104307

\section{INTRODUCTION}

Wettability properties inside a porous medium can be of crucial importance in many applications such as water-gas transport within the gas diffusion layer (GDL) of proton exchange membrane fuel cells (PEMFC) [1,2] or fluid displacement in geological media encountered in petroleum recovery [3]; they widely control the behavior of powder-based materials [4] and the performance of packedbed reactors [5].

As discussed and analyzed for instance in Refs. [6-8], the change in wettability conditions can dramatically modify the liquid water pattern from a capillary fingering pattern to a compact pattern as the wettability properties change from hydrophobic to hydrophilic. As pointed out in Ref. [9], capillary fingering allows maintaining a significant fraction of the pore space free of liquid water and thus available for the gas transport through a GDL substrate for instance, whereas a compact pattern blocks the gas access and is thus detrimental to the PEMFC performance. Methods for characterizing the wettability of a porous substrate generally lead, at best, to the characterization of an "average" or apparent contact angle. A traditional technique developed for core analysis in the oil industry consists of the determination of a wettability index, obtained from the comparison of saturations obtained from spontaneous and forced displacements, the so-called Amott index [10] or U.S. Bureau of Mines (USBM) index [11]. However, these indexes are sensitive to experimental details in practice and remain difficult to correlate (see a discussion in Ref. [12]). Nuclear magnetic resonance techniques have also been devised to infer the wettability properties of a porous material from the proton lattice relaxation time [13].

An alternative somewhat popular method is the sessile drop method [14]. It consists of placing a droplet of liquid water at the surface. The contact angle can then be measured by processing side

*didier.lasseux@u-bordeaux.fr 
images of the droplet. Nevertheless, the interpretation is far from straightforward when the droplet is placed on a porous surface, especially in the case of fibers of heterogeneous wettability. Moreover, wettability at the surface is not necessarily representative of that inside the porous medium.

Another commonly used method is the Washburn method [15-17]. It consists of measuring the liquid sorption kinetics into the sample, first with a liquid of very low contact angle and second with the liquid of interest for the application under consideration. Obviously, the method cannot be directly applied with water and a hydrophobic material since there is no sorption in this case. However, as shown in Ref. [16], the Washburn method can be combined with the Owens-Wendt two-parameters theory [18] in order to estimate the internal contact angle for water in a hydrophobic porous material. Nevertheless, as pointed out in Ref. [16], the Washburn method only allows estimating an apparent or average contact angle reflecting a statistical average of the porous material wettability properties at the pore scale.

More detailed information on the wettability properties at the pore scale can be obtained from scanning electron microscopy (SEM) images of droplets or menisci inside the pore space. The measurement of local contact angles from images is however very difficult since droplets are threedimensional objects $[19,20]$. Some issues and perspectives in the wettability characterization of porous materials were recently discussed in Ref. [21].

In the present paper, a different approach from those reported so far is developed in order to obtain the internal contact angle distribution, in an average or statistical sense, inside the pore space of the material. The method is motivated by two observations. First, 3D digital images of porous microstructures can now be obtained without too many difficulties, using either x-ray microtomography techniques [22,23] or others such as focused ion-beam SEM [24], depending on whether the pore size is in the micrometer or submicrometer range. For instance, 3D images of GDL substrate microstructures were obtained by x-ray tomographic microscopy [23] and it can thus be reasonably assumed that $3 \mathrm{D}$ digital images of porous microstructures of interest are available. Second, macroscopic properties, such as the capillary pressure or relative permeability vs saturation curves, are sensitive to the internal wettability conditions $[6,25]$. Consequently, the question arises as to whether this sensitivity can be exploited to characterize the internal wettability conditions. In other words, the idea here is to explore the possibility of characterizing the internal wettability conditions from digital images of the porous microstructure together with the capillary pressure and relative permeability curves. This characterization is addressed as an inverse optimization problem, which includes two-phase flow simulations within the microstructure and an optimization procedure combining a genetic algorithm [26,27] and the hill-climbing algorithm [26,28]. This type of approach has shown promising results for the identification of the pore size distribution from macroscopic flow data such as the capillary-pressure curve and/or normalized flow curves [29]. Contrary to this previously reported work where the objective was to identify the pore size distribution assuming a spatially uniform wettability throughout the pore space, the objective here is to identify the internal contact angle spatial distribution, knowing the microstructure geometry and thus the pore size distribution. As in the above-mentioned work, pore network models (PNM) $[30,31]$ are used for the two-phase flow simulations in the pore space. This approach is preferred to direct two-phase flow simulations (see, e.g., Refs. [32,33]) because of its efficiency in terms of computational requirements. The PNM simulations require identifying the network from the microstructure digital images. Various algorithms are available for this purpose as reported in the literature (see for instance Refs. [23,34,35] and references therein). However, since the focus is on the development of a new characterization methodology, a somewhat simplified version of the internal wettability characterization problem is considered in what follows. First, the network is a given two-dimensional square network so as to speed up the simulations (compared to the typical unstructured 3D network extracted from a microstructure 3D digital image). Second, the internal contact angle of the liquid on the solid surface in the presence of the gas phase can take two values, either $80^{\circ}$ or $115^{\circ}$. This choice is inspired from what can be encountered in a GDL whose substrate is made of graphitized carbon fibers that is further coated with polytetrafluoroethylene (PTFE). The two values of the contact angle respectively correspond to that of water in the presence of vapor on graphite and on PTFE flat surfaces under static conditions [36]. Further details on the model problem 


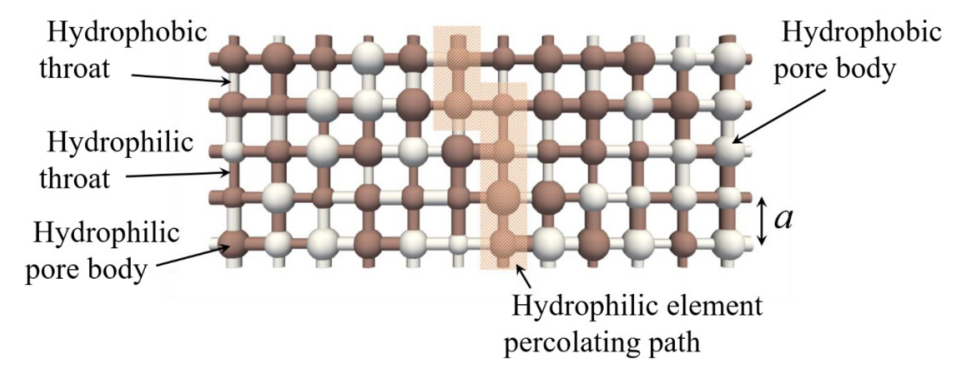

FIG. 1. A $12 \times 5$ square pore network. Throats and pore bodies in brown are hydrophilic $\left(\theta=80^{\circ}\right)$. Throats and pore bodies in gray are hydrophobic $\left(\theta=115^{\circ}\right)$. A hydrophilic element percolating path, connecting the network inlet (at the bottom) to outlet (at the top), is illustrated.

under consideration are given in the next section. The purpose is to identify the wettability properties of the medium in an average sense, i.e., the hydrophilic pore fraction. Indeed, there is obviously not a unique solution in terms of this spatial local distribution that characterizes a medium featuring the same capillary pressure and relative permeability curves as these data do not contain the necessary information that constrains the problem to retrieve an unequivocal link of this local property to these two macroscopic characteristics.

The present article is organized as follows. In Sec. II, the model porous medium, a twodimensional square pore network of pore bodies and throats, is presented. The algorithms used to simulate the displacement on the network and compute the capillary pressure and relative permeability curves are presented in Sec. III. The inverse optimization method for determining the local contact angle distribution in the network is described in Sec. IV. Section V is dedicated to results obtained with the optimization method. A short discussion is presented in Sec. VI. Conclusions are drawn in Sec. VII.

\section{MODEL POROUS MEDIUM}

To simplify the presentation and assess the capability of the method of local contact angle characterization, a two-dimensional square pore network is considered. As illustrated in Fig. 1, the pore space is an assembly of two types of elements: pore bodies and throats. Pore bodies are spheres of radius $r_{p}$ located at the nodes of a regular square grid. The distance between two adjacent nodes is the lattice spacing, $a$, with $a=350 \mu \mathrm{m}$ in all the simulations presented below. The size of the network is the number of nodes (pore bodies) in each direction (for example, Fig. 1 shows a $12 \times 5$ square network). Throats are cylindrical channels of radius $r_{t}$. The throat and pore body sizes are randomly distributed according to a given probability density function (pdf). In what follows, a uniform pdf is considered with $r_{t}$ varying between $r_{t \text { min }}=20 \mu \mathrm{m}$ and $r_{t \max }=40 \mu \mathrm{m}$. Similarly, the pore body sizes are distributed according to a uniform pdf with the constraint that a pore body size is equal to or greater than the largest throat to which the pore body under consideration is connected. In this work, the minimum and maximum pore body sizes are, respectively, $r_{p \min }=80 \mu \mathrm{m}$ and $r_{p \max }=120 \mu \mathrm{m}$. The model of wettability heterogeneity consists of assuming that a fraction, $f$, of the pore bodies and throats in the network is hydrophilic, with a contact angle $\theta=80^{\circ}$, whereas the complementary fraction is hydrophobic with $\theta=115^{\circ}$. Thus, a fully hydrophobic (hydrophilic, respectively) network corresponds to $f=0$ ( $f=1$, respectively). Networks of mixed wettability correspond to intermediate values of $f(0<f<1)$. Hydrophilic elements are randomly selected in the network until $f$ reaches the desired value.

\section{CAPILLARY PRESSURE AND RELATIVE PERMEABILITY CURVES}

As will be detailed in the next sections, the capillary pressure and liquid relative permeability curves during liquid invasion of the network initially saturated by the gas phase are the key 
properties of the porous medium on which the identification of the wettability properties proposed in this work relies. It is hence of importance to first provide the procedure to determine these quantities for a given network.

\section{A. Local invasion rules}

The PNM computation of the capillary pressure and relative permeability curves is based on the definition of local invasion rules. An invasion capillary-pressure threshold (ICPT) is associated with each element, pore body or throat, in the network. The ICPT of a throat is simply given by the Young-Laplace equation as

$$
p_{c}=\frac{-2 \gamma \cos (\theta)}{r_{t}},
$$

where $\gamma$ is the interfacial tension between the two fluids. Similarly, the ICPT of a hydrophobic pore body is given by

$$
p_{c}=\frac{-2 \gamma \cos (\theta)}{r_{p}} .
$$

As first shown in Ref. [37], the situation is more involved for a hydrophilic pore body. In fact, the invasion in that case depends on the number of adjacent hydrophilic throats already filled with liquid. This aspect can be taken into account by defining the ICPT of a hydrophilic pore body as [38]

$$
p_{c}=\frac{-2 \gamma \cos (\theta)\left[1+0.25\left(N_{\text {neighbors }}-1\right)\right]}{r_{p}},
$$

where $1 \leqslant N_{\text {neighbors }} \leqslant N_{c}-1, N_{\text {neighbors }}$ being the number of hydrophilic adjacent throats already containing liquid water and $N_{c}$ the connectivity of the network, i.e., the number of throats connected to a pore body (here, $N_{c}=4$ ).

If a meniscus is located at the entrance of a pore network element of ICPT $p_{c}$, it can move into the element only when $P_{w}-P_{g}>p_{c}$, where $P_{w}$ and $P_{g}$ are, respectively, the pressures in the liquid and the gas phase.

\section{B. Capillary-pressure curve computation}

The capillary-pressure curve $P_{c}(S)$, where $S$ is the saturation of the displaced fluid, is computed using an algorithm similar to the one presented in Refs. [29,39], but takes into account here the presence of hydrophilic elements. This algorithm mimics the commonly used experimental procedure [25]. The network is initially fully saturated by the gas phase. Then, liquid water is injected into the network through a sequence of pressure steps. A membrane is supposed to be present at the outlet so that only the displaced fluid can exit the network. The capillary pressure is determined as a function of $S$ for successive states of hydrostatic equilibrium corresponding to small increment, $d P_{w}$, of the liquid water pressure (the gas-phase pressure, $P_{g}$, is kept constant). The capillary pressure is by definition $P_{c}=P_{w}-P_{g}$. The algorithm used to determine the saturation evolution right after a pressure increment can be summarized as follows:

(i) Initialization of a menisci position vector that stores all interfacial pore bodies and throats. A pore body or throat is interfacial when it is occupied by the displaced fluid (gas phase in our simulation) and is adjacent to an element, pore body or throat, containing the displacing fluid (liquid water).

(ii) Identification of the interfacial pore body or throat to be invaded first: it corresponds to the interfacial pore body or throat featuring the minimum ICPT among the interfacial pore bodies and throats having an ICPT smaller than the imposed pressure difference, $P_{w}-P_{g}$, between the two fluids. The ICPT values are obtained from Eqs. (1)-(3). For hydrophilic pore bodies or throats connected to liquid-gas interfaces, the ICPT is negative $\left(\theta=80^{\circ}<90^{\circ}\right)$. Hence, they are the ones to be selected 
first. In addition, since $r_{p}>r_{t}$, the ICPT associated with a hydrophilic throat is smaller than the hydrophilic pore bodies' ICPT, which means that hydrophilic throats are more likely to be selected. However, for some cases where there exists a hydrophilic pore body connected to many hydrophilic throats, the ICPT can be smaller as indicated by Eq. (3).

(iii) Invasion of the pore body or throat selected in the previous step; the vector of menisci positions is updated: interface positions are either added or deleted according to the pore body or throat invaded.

(iv) Steps (ii) and (iii) are repeated until no further interfacial pore body or throat satisfying the condition ICPT $<P_{w}-P_{g}$ can be identified.

(v) Computation of the overall gas saturation, $S$.

For simplicity, trapping $[8,40]$ is neglected. This means that the displaced fluid in a network element, pore body or throat, is implicitly assumed to be always connected to the outlet face and can thus be always displaced when the corresponding capillary-pressure invasion threshold is overcome.

The impact of the hydrophilic elements fraction on the fluid distribution at breakthrough (the breakthrough is when the displacing fluid becomes connected to the outlet) is illustrated in Fig. 2 keeping the same pore network structure.

A capillary fingering pattern is observed for $f \sim 0$ as expected for a quasistatic drainage process. A compact pattern is obtained for $f \sim 1$. The pattern obtained for $f \sim 0.4$ resembles the capillary fingering pattern but actually results from the fact that the percolating subnetwork formed by the hydrophilic pore bodies and throats is a percolation cluster. An invasion percolation cluster $(f \sim 0)$ is about the same fractal object as the hydrophilic elements percolation cluster $(f \sim 0.4)$. This is why both cases $(f \sim 0$ and $f \sim 0.4$ ) lead to the same type of phase distribution despite the change in the local mechanisms controlling the growth of the liquid-gas interface in the hydrophobic regions and the hydrophilic ones, respectively.

The capillary pressure curves obtained for one realization of a $30 \times 30$ network with the above algorithm when the fraction, $f$, of hydrophilic elements in the network is varied is reported in Fig. 3. As mentioned before, the pore body and throat sizes are distributed according to uniform pdf with $r_{t \text { min }}=20 \mu \mathrm{m}, r_{t \text { max }}=40 \mu \mathrm{m}, r_{p \min }=80 \mu \mathrm{m}, r_{p \max }=120 \mu \mathrm{m}$, and $a=350 \mu \mathrm{m}$.

This figure illustrates the sensitivity of the capillary-pressure curve to the hydrophilic elements fraction $f$, showing a very significant impact of the local contact angle distribution on $P_{c}(S)$ at the macroscopic level. This originates from the dependence of the fluid distribution on the hydrophilic elements fraction illustrated in Fig. 2. Based on a previous work [6], $f=0.5$ approximately corresponds to the hydrophilic elements percolation threshold, $f_{c}$, i.e., the minimum value of $f$ for which the hydrophilic elements form a cluster spanning the network. However, it can be observed that the value $f_{c}=0.4$, as noticed in the discussion on the patterns reported in Fig. 2, is possible for a particular realization. This difference is due to finite size effects. Another important feature, as can be observed from Fig. 3, is that the sensitivity of the capillary-pressure curve to $f$ is larger for $f>f_{c}$ than for $f<f_{c}$. This is consistent with the patterns depicted in Fig. 2, which shows that the transition from a fingering pattern to a compact pattern occurs for $f>f_{c}$

\section{Relative permeability curve}

As in Ref. [29], only the relative permeability for the invading (liquid) phase is computed and the reader is referred to this article for the reasons motivating this choice. An equilibrium liquid gas distribution in the network is obtained for each value of the capillary pressure, $P_{c}$, under consideration. The invading phase relative permeability is determined from the computation of the invading fluid viscous flow through the network for each equilibrium phase distribution, that is for each point of the capillary-pressure curve. The computation of the displacing fluid flow rate, $Q_{i}$, is performed as follows [29,39]. At each saturation equilibrium, $i$, a pressure difference, $\Delta P$, much smaller than the capillary pressure characterizing this equilibrium, is applied in the displacing fluid between the network inlet and outlet. For the resulting flow, the mass conservation equation is 


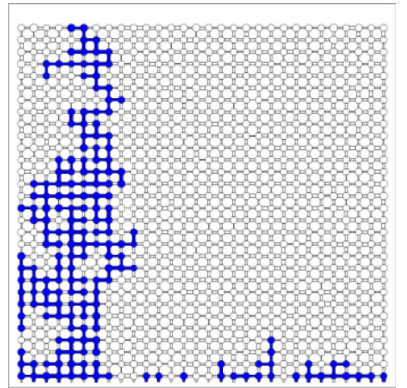

(a)

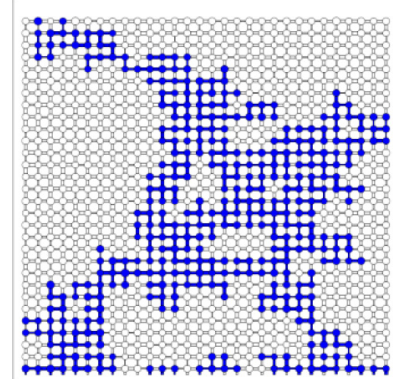

(d)

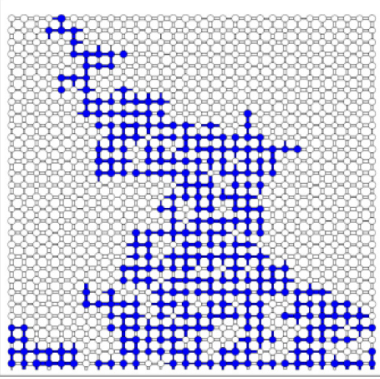

(g)

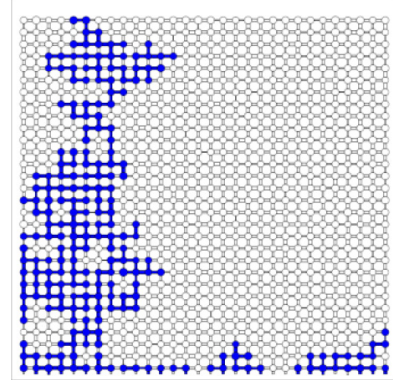

(b)

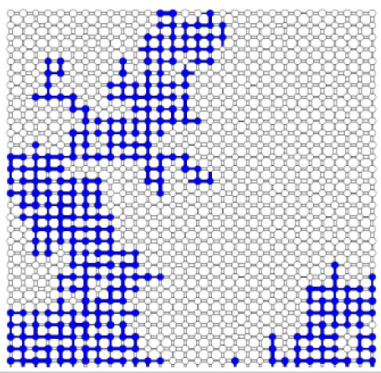

(e)

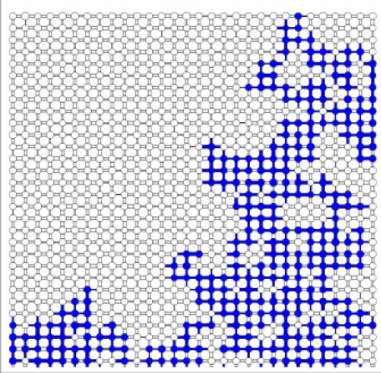

(h)

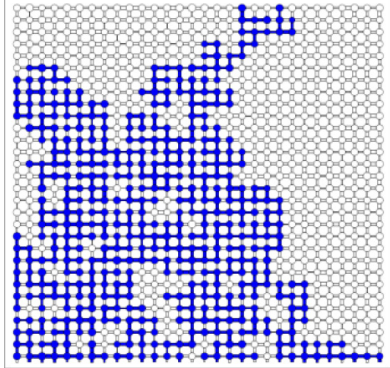

(c)

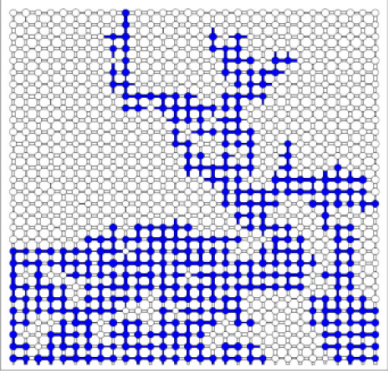

(f)

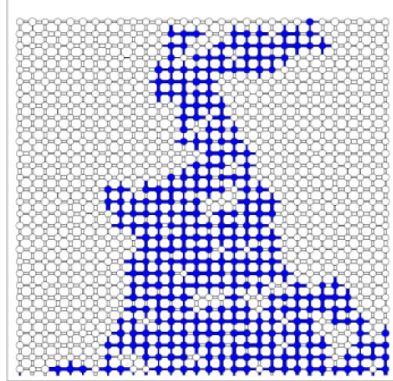

(i)

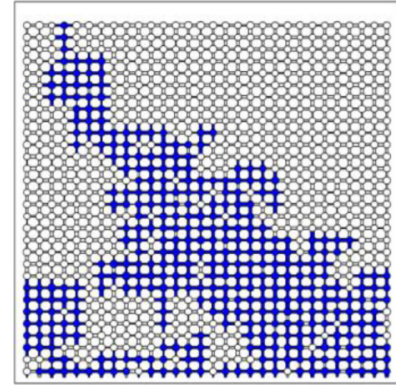

(j)

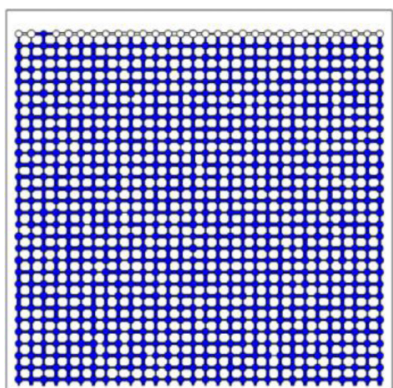

(k)

FIG. 2. Fluid distribution for various values of the hydrophilic pore body and throat fraction, $f$, in a $2 \mathrm{D}$ $30 \times 30$ square network. (a) $f=0$; (b) $f=0.1$; (c) $f=0.2$; (d) $f=0.3$; (e) $f=0.4$; (f) $f=0.5$; (g) $f=0.6$; (h) $f=0.7$; (i) $f=0.8$; (j) $f=0.9$; and (k) $f=1$. The displacing fluid (in blue) is injected from the bottom using the capillary-pressure algorithm. The displaced fluid (in gray) escapes from the top. Patterns correspond to fluids distributions at breakthrough. 


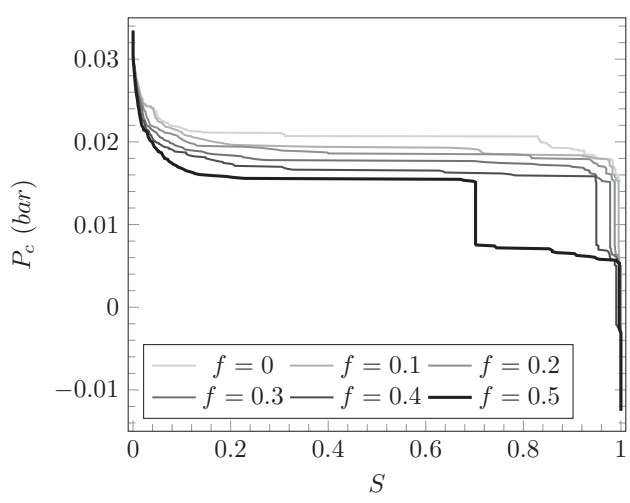

(a)

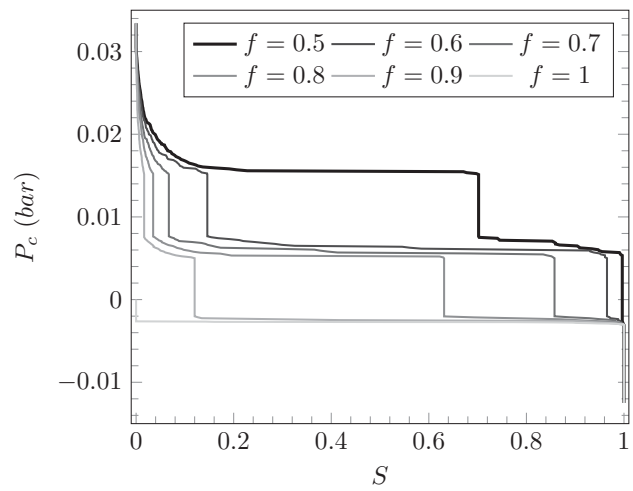

(b)

FIG. 3. Capillary-pressure curves computed for a $30 \times 30$ network for several values of the fraction, $f$, of hydrophilic elements in the network. The curve corresponding to $f=0.5$ is represented with a thicker black line. (a) The top curve corresponds to $f=0$ (fully hydrophobic network). (b) The bottom curve corresponds to $f=1$ (fully hydrophilic network).

expressed at each invaded pore body $j$, yielding

$$
\sum_{k} Q_{i-j, k}=0
$$

where $Q_{i-j, k}$ is the displacing fluid volume flow rate between pore body $j$ and adjacent pore body $k$. If the throat between pore bodies $j$ and $k$ is occupied by the displaced fluid, then $Q_{i-j, k}=0$. If the throat is occupied by the displacing fluid, then

$$
Q_{i-j, k}=\frac{\pi r_{t-j, k}^{4}}{8 \mu l_{j, k}} \Delta P_{j, k},
$$

where $r_{t-j, k}$ and $l_{j, k}$ are the radius and length of the throat linking the two pore bodies, respectively, $\mu$ is the displacing fluid dynamic viscosity, while $\Delta P_{j, k}$ is the pressure drop in the displacing fluid between the two neighboring pore bodies. Taking into account the pressure boundary conditions at the inlet $\left(P_{\text {inlet }}=\Delta P+P_{\text {outlet }}\right)$ and outlet $\left(P_{\text {outlet }}=\right.$ Const $)$ together with the zero-flux condition imposed on the lateral faces of the network, Eqs. (4) and (5) lead to a linear system for the pressure field in the invading fluid. This system is numerically solved using the conjugate gradient method. Once the pressure field is obtained, $Q_{i}$ can be computed at the inlet using Poiseuille's law applied to all displacing fluid throats connected to the inlet. Then, the generalized Darcy law is applied to determine the displacing fluid relative permeability,

$$
Q_{i}=A \frac{K K_{r}\left(P_{c}\right)}{\mu} \frac{\Delta P}{L},
$$

where $P_{c}$ is the capillary pressure corresponding to the saturation equilibrium $i, A$ is the network cross-section surface area, $L$ is the network length, $K$ is the porous medium intrinsic permeability, and $K_{r}$ is the nonwetting fluid relative permeability. From Eq. (6), the relative permeability is computed as

$$
K_{r}\left(P_{c}\right)=\frac{Q_{i}}{Q_{\max }}
$$

where $Q_{\max }=A \frac{K}{\mu} \frac{\Delta P}{L}$ is the displacing fluid flow rate when the network is fully saturated by the displacing fluid and a pressure difference $\Delta P$ is applied between the inlet and outlet. 


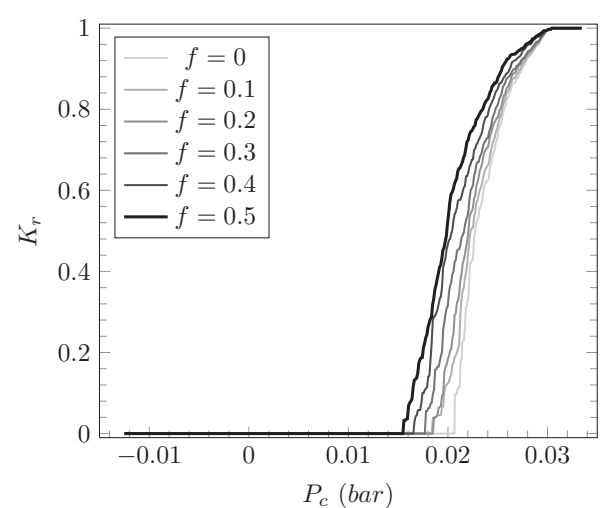

(a)

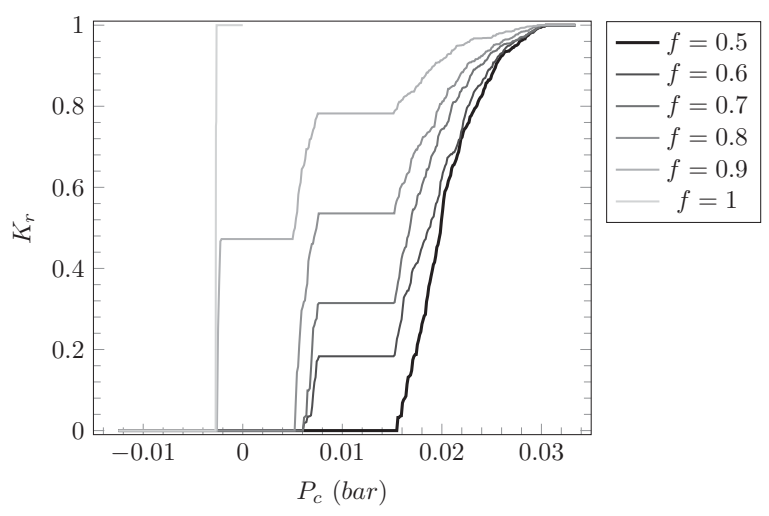

(b)

FIG. 4. Displacing fluid relative permeability curves computed for the same $30 \times 30$ network as for Fig. 3 for several values of the fraction $f$ of hydrophilic elements in the network. The curve at the far right in the figure on the left corresponds to $f=0$ (fully hydrophobic network). The curve at the far left in the figure on the right corresponds to $f=1$ (fully hydrophilic network). The curve corresponding to $f \approx 0.5$ is represented with a thicker black line.

In Fig. 4, the displacing fluid relative permeability, obtained with the above algorithm, is represented versus the capillary pressure for the fraction $f$ of hydrophilic elements in the network varying from 0 to 1 (with an increment of 0.1 ) for the same $30 \times 30$ network as for the capillary-pressure curves computation (Fig. 3).

As for the capillary-pressure curves (Fig. 3), Fig. 4 illustrates the noticeable sensitivity of the displacing fluid relative permeability to the hydrophilic elements fraction $f$. Again, it can be noted that sensitivity to $f$ is greater for $f>f_{c}(\sim 0.4)$ but still significant at all the values of $f$ investigated here. How this sensitivity is exploited is described in the next section.

\section{LOCAL CONTACT ANGLE DISTRIBUTION AS AN INVERSE OPTIMIZATION PROBLEM}

The problem of determining the distribution of the local contact angle in the network is addressed as an inverse optimization problem. The problem can be expressed as follows. Knowing the $K_{r}\left(P_{c}\right)$ and $P_{c}(S)$ curves for a given network, can the local contact angle distribution in the network be predicted? In this context, the direct problem is of course to compute the $K_{r}\left(P_{c}\right)$ and $P_{c}(S)$ curves of the network under consideration. As for the direct problem (see Sec. III), the inverse problem is solved using PNM.

To build and test the procedure, a $30 \times 30$ square network is generated with a specified value of the fraction, $f$, of hydrophilic elements. The structure is that described in Sec. II: pore body and throat sizes are randomly chosen according to a uniform distribution with $r_{p \min }=80 \mu \mathrm{m}, r_{p \max }=$ $120 \mu \mathrm{m}$ for the former and $r_{t \min }=20 \mu \mathrm{m}, r_{t \max }=40 \mu \mathrm{m}$ for the latter. The lattice spacing is $a=350 \mu \mathrm{m}$. This network is referred to as the reference network. It shall be viewed as the porous medium to be characterized for which the experimental measurement of $Q_{i}\left(P_{c}\right)$ and $P_{c}(S)$ would have been performed in practice, and which local contact angle distribution is to be determined. Since the local contact angle distribution considered in our model problem is bivalued, a wettability label, 0 or 1, is assigned to each pore network element. The label is 1 when the element is hydrophilic $\left(\theta=80^{\circ}\right)$ and 0 when the element is hydrophobic $\left(\theta=115^{\circ}\right)$.

The inverse problem is solved using an optimization method based on a searching algorithm, which is a hybrid version of the genetic and hill-climbing algorithms [26-28]. The genetic algorithm is a searching algorithm based on the biological evolution laws, including reproduction, mutation, and natural selection. A population of individuals is first defined. In our case, the individuals are 
realizations of the pore network, that is, random spatial distributions of the wettability label in the network (for a given fraction $f$ of hydrophilic elements). Every individual has genes. A gene in the pore network is the element wettability label. The aim of the algorithm is to identify the hydrophilic elements fraction, $f$, via the minimization of a fitness function, which value is estimated from a direct solution for each individual. The fitness function, denoted by $\phi(\boldsymbol{w})$, is an objective function comparing the $K_{r}\left(P_{c}\right)$ and $P_{c}(S)$ curves obtained by direct simulation for the individuals to the reference ones. Here, $\boldsymbol{w}$ is a vector which components are the wettability labels assigned to each element, pore body or throat, in a network. The fitness function is defined as

$$
\Phi(\boldsymbol{w})=\sum_{j=1}^{n}\left|K_{r n-\operatorname{ref} j}-K_{r n-\mathrm{ind} j}(\boldsymbol{w})\right|+\sum_{j=1}^{n}\left|S_{\text {ref } j}-S_{\text {ind } j}(\boldsymbol{w})\right|,
$$

where $n$ is the total number of pressure steps, $K_{r n-\operatorname{ref} j}$ and $K_{r n-\text { ind } j}$ are the reference network and individual relative permeabilities corresponding to the $j$ th pressure increment, respectively; $S_{\text {ref } j}$ and $S_{\text {ind } j}$ are the reference and individual saturations at the $j$ th pressure increment.

The main steps of the algorithm can be summarized as follows.

The first step consists of generating a series of $N_{0}$ parent realizations ( $N_{0}=16$ in this work) of various hydrophilic elements fraction, $f$, to which evolutionary laws and natural selection are applied. Since the hydrophilic elements fraction is the unknown to be determined, this fraction is varied between 0 and 1 over the $N_{0}$ parent realizations.

The relative permeability and capillary-pressure curves, together with the corresponding fitness function, are computed for each individual parent. After this initialization procedure, the genetic algorithm is executed according to the following steps:

(i) The natural selection is performed by randomly choosing a subset of $N_{1}$ individuals among the generation of individuals (here, $N_{1}=5$ ). The two corresponding to the best fitness values are selected.

(ii) Two evolutionary operators, namely crossover and mutation, are applied to the two parent individuals. Crossover is the process by which the two selected parent individuals give birth to a child realization by exchanging randomly wettability labels (genes). Mutation is an operation applied separately to both selected parent realizations. It consists of altering genes (the wettability labels). Mutations are generally supposed to happen rarely. In the present case, the mutation rate is taken equal to 0.01 . This means, for example for a $30 \times 30$ network containing 2640 elements (900 pore bodies and 1740 throats), that 9 pore bodies and 17 throats are selected randomly. Then, the wettability labels of these 26 elements are specified randomly.

(iii) The $K_{r}\left(P_{c}\right)$ and $P_{c}(S)$ curves and the fitness values for the two children are computed and, among the four individuals (the two parents and the two children), the two individuals having the best fitness values are selected. They are kept to form the new generation.

(iv) Steps (i) to (iii) are repeated until there are no more individuals in the parent population and a new generation of $N_{0}$ individuals is formed.

(v) The process made of steps (i) to (iv) is iterated until convergence is reached. By convergence, it is meant that the fitness of all the individuals from one generation to the following one does not change by more than about $0.1 \%$.

For all the cases under study in this work, 200 generations (iterations) were enough to reach convergence at which the solution is estimated to be close to the global solution. Then, a new step is performed using the hill-climbing algorithm.

The objective of the hill-climbing algorithm, which, like the genetic algorithm is a searching algorithm, is to gradually modify the wettability distribution within a single individual so as to refine the convergence toward the reference network wettability distribution. To this end, the hillclimbing algorithm is operated on the best-fitted individual in the population obtained at the end of genetic algorithm execution. The procedure is the following. An element is randomly selected in the network. The wettability of this element is changed (from hydrophobic to hydrophilic if this element is hydrophobic or conversely). The fitness function is then computed. If this results in a 


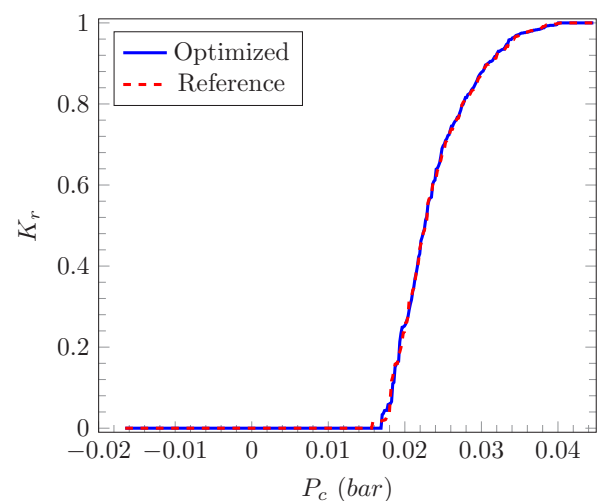

(a)

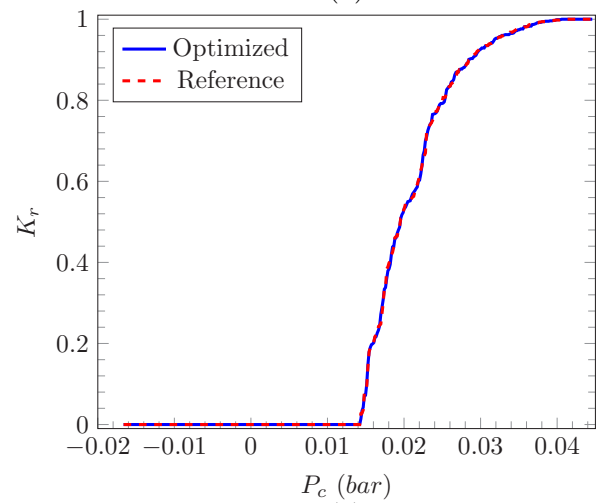

(c)

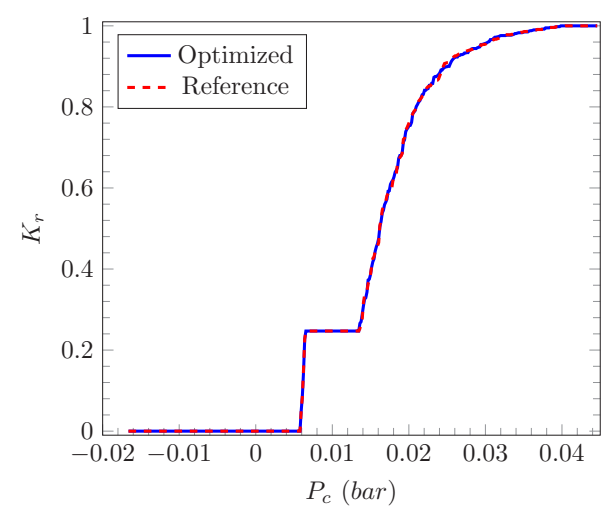

(e)

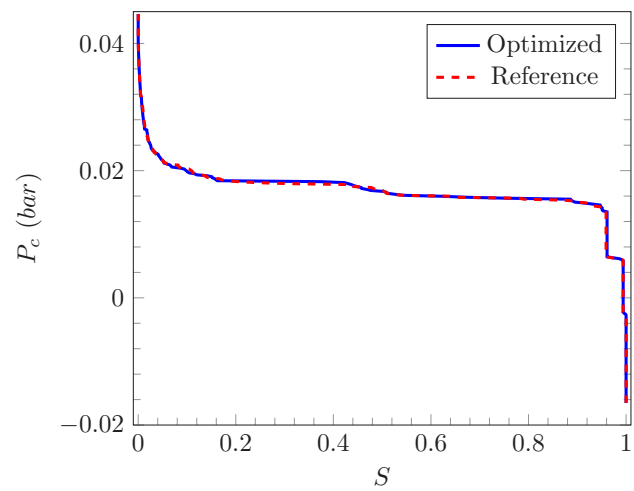

(b)

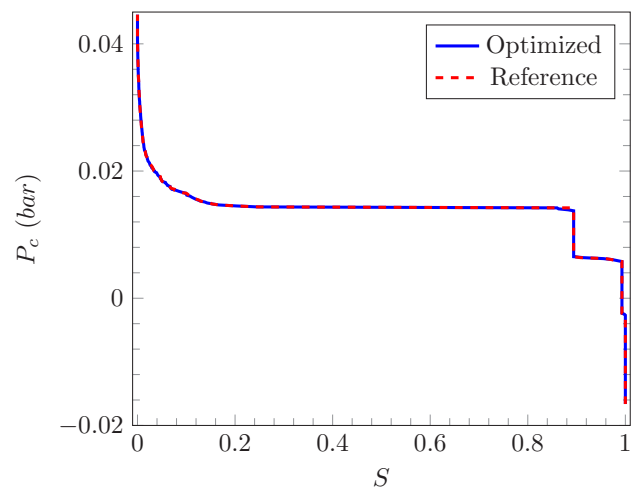

(d)

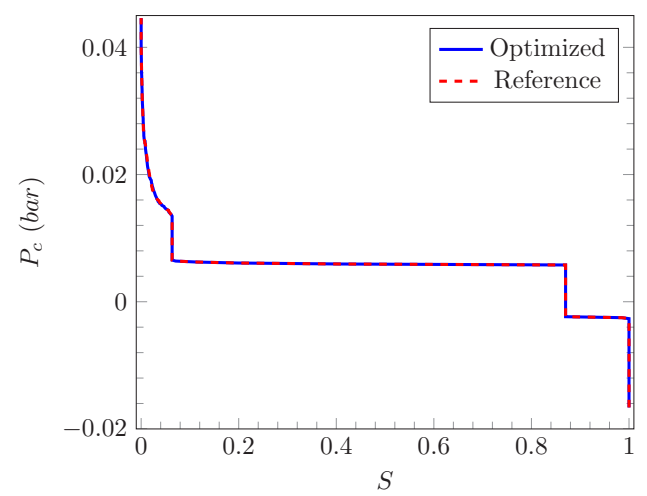

(f)

FIG. 5. Comparison between the relative permeability curves (left column) and capillary-pressure curves (right column) of the reference and optimized networks. (a), (b) $f=30 \%$; (c), (d) $f=50 \%$; and (e), (f) $f=70 \%$.

decrease of the value of the fitness function, then a small number (randomly chosen between 1 and 5) of hydrophobic elements are randomly selected among the hydrophobic elements and they are changed into hydrophilic elements. This operation is repeated until the fitness function does not decrease. The whole hill-climbing algorithm procedure is then repeated over and over again until convergence, which is considered to be reached when the value of the fitness function does not change by more than $0.1 \%$ over 20 successive iterations of the hill-climbing algorithm. At this 
TABLE I. Comparison between the fraction of hydrophilic elements in the reference network, $f$, and the value, $f_{o}$, identified with the optimization procedure.

\begin{tabular}{lccc}
\hline \hline $\begin{array}{l}f(\%) \\
\text { (Reference network) }\end{array}$ & $\begin{array}{c}f_{o}(\%) \\
\text { (Optimization procedure) }\end{array}$ & $\begin{array}{c}\text { Error }(\%) \\
\left|f_{o}-f\right|\end{array}$ & $\begin{array}{c}\text { Relative error }(\%) \\
\frac{\left|f_{o}-f\right|}{f}\end{array}$ \\
\hline 10 & 11.59 & 1.59 & 15.9 \\
20 & 22.74 & 2.74 & 13.7 \\
30 & 29.7 & 0.3 & 1 \\
40 & 38.07 & 1.93 & 4.81 \\
50 & 51.78 & 1.78 & 3.56 \\
60 & 59 & 1 & 1.66 \\
70 & 68.56 & 1.44 & 2.06 \\
80 & 79.74 & 0.26 & 0.33 \\
90 & 89.15 & 0.85 & 0.94 \\
\hline \hline
\end{tabular}

point, the optimized value of $f$ is denoted $f_{o}$. and the corresponding network is referred to as the optimized network.

\section{RESULTS}

Tests were first performed for three values of the hydrophilic elements fraction in a $30 \times 30$ reference network, namely $f=30,50$, and $70 \%$. The comparison between the relative permeability and capillary-pressure curves of the reference and optimized networks is reported in Fig. 5.

As can be observed, the optimization procedure leads to an excellent agreement between the curves for the reference and optimized networks for the three values of $f$.

A similar agreement was obtained for other values of $f$ over the whole range of $f$ in the interval $f=10 \%-f=90 \%$. The results on $f_{o}$ over this interval are reported in Table I, showing that the optimization procedure allows retrieving the fraction of hydrophilic elements with a relative error less than $16 \%$ on the whole range of $f$. This consistently confirms the excellent agreement on the relative permeability and capillary-pressure curves depicted in Fig. 5.

The performance of the optimization procedure is further illustrated in Fig. 6, where the distribution of hydrophilic and hydrophobic elements in the reference and optimized networks are compared. Of course, it is not expected that the optimization procedure leads to a one-to-one matching regarding the localization of the hydrophilic elements in the reference and optimized networks. The quality of the matching must be understood in a statistical sense. In other words, the matching is satisfactory if the reference and optimized networks can be considered as two realizations of the random process consisting here of distributing randomly the hydrophilic elements in the network. Therefore, it should be clear that the solutions on the local distribution of the wettability for the optimized network depicted in Fig. 6 are not unique. Repeating the optimization procedure typically leads to the same value for $f$ but generally to a different realization of the optimized network. It is certainly possible to go deeper in a statistical analysis by comparing more advanced statistical properties than simply the average, that is $f$. For instance, it could be tempting to make use of the Minkowski functionals [41]. However, a detailed study of the statistics is left for a more in-depth investigation in a future work. As such, the prediction of the fraction of hydrophilic elements, $f$, is already a quite remarkable and interesting achievement representing a step forward in the characterization of wettability properties of a porous material.

\section{DISCUSSION}

Two macroscopic classical data, namely the displacing fluid relative permeability and the capillary-pressure curves, were considered as input data for the optimization procedure to identify 


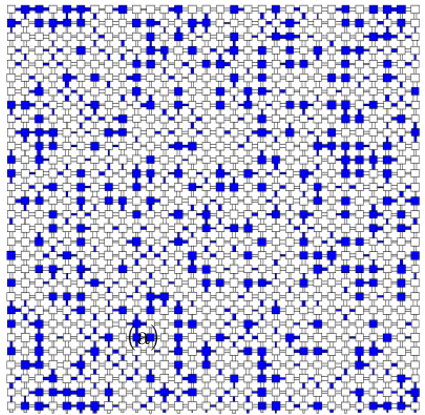

(a)

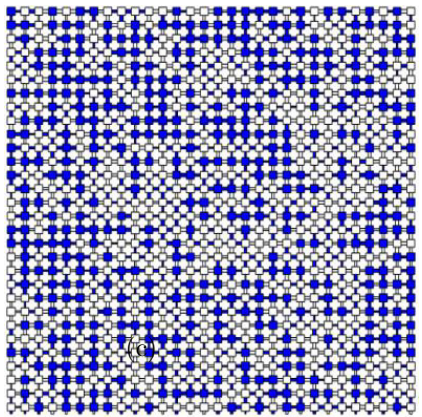

(c)

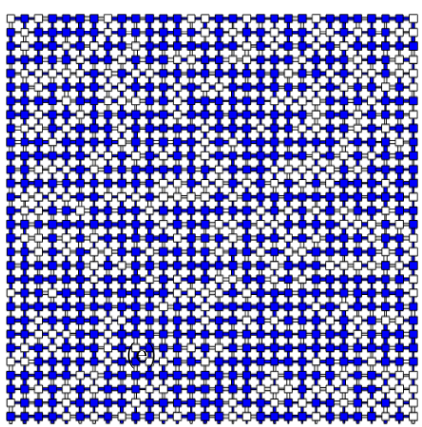

(e)

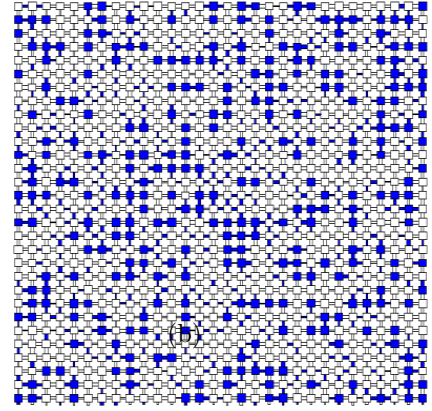

(b)

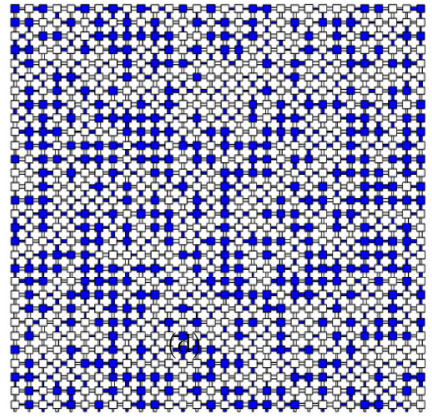

(d)

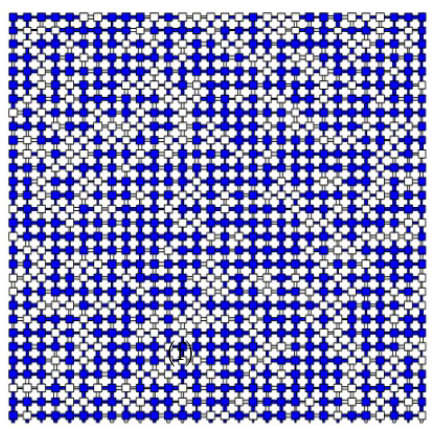

(f)

FIG. 6. Distribution of hydrophilic (in blue) and hydrophobic (in light gray) elements in the reference network (left column) and optimized network (right column). (a), (b) $f=30 \%$; (c), (d) $f=50 \%$; and (e), (f) $f=70 \%$.

the hydrophilic elements fraction. As considered in Ref. [29] in the case of the throat size distribution (TSD) determination problem, it is possible to perform the optimization procedure considering only one datum, either the capillary-pressure curve or the relative permeability curve. For the TSD problem, it was found that considering both data led to the best results and that the consideration of the capillary-pressure curve alone led to better results than the relative permeability curve alone. For this reason, both data were considered in the present paper. Nevertheless, it would be interesting to test the quality of the results when, for instance, the capillary-pressure curve alone is considered. Conversely, it can be expected that considering more macroscopic data, such as for instance the displaced fluid relative permeability in addition to the displacing fluid relative permeability and the capillary pressure should improve the results. 
The optimization procedure was investigated here considering a 2D network. The question arises as to whether similar results can be expected with 3D networks. Some indications on this point can be obtained from the PNM simulation results presented in Ref. [6], where computations of the capillary pressure and relative permeability are presented for a 3D cubic network featuring various fraction of hydrophilic pores. A crucial point is of course that the macroscopic data, i.e., the capillary-pressure curve and/or the relative permeability curve are sensitive to the wettability variations. In this respect, it can be observed from Figs. 3 and 4 that the curves sensitivity to $f$ is more significant for $f>40 \%$ than for $f<40 \%$. A similar observation can be made regarding the $3 \mathrm{D}$ simulation results presented in Ref. [6] where a critical value $f_{c}\left(f_{c} \sim 0.48\right)$ was introduced. Furthermore, in this reported work, the sensitivity to $f$ was still less pronounced for $f<f_{c}$ than for the case considered in the present paper. This is an indication that the local wettability characterization method could be less accurate for $f<f_{c}$ than for $f>f_{c}$. This is consistent with the results reported in Table I showing a somewhat greater relative error for $f<0.4$. However, it can be noticed from Table I that the predictions of $f$ are still very satisfactory in the range $f<0.4$ where sensitivity decreases. As discussed in some detail in Ref. [6], $f_{c}$ corresponds to the network percolation threshold [42]. In the context of percolation theory, the distribution of the hydrophilic elements considered in the present work is a mixed bond-site percolation problem since the wettability of both the throats (bonds) and pore bodies (sites) is varied. The percolation threshold, $f_{c}$, corresponds to the smallest fraction of hydrophilic elements for which a percolating path of hydrophilic elements exists between the network inlet and outlet. According to percolation theory [42], the greater the coordination, i.e., the number of pore bodies to which a pore body is connected, the lower is the network percolation threshold. The coordination number in real porous media can be expected to be greater than that of the square network considered in the present study (i.e., 4) or the cubic network considered in Ref. [6] (i.e., 6). In other words, the method of characterization proposed here can thus be expected to be accurate over the full range of $f$ when applied to a real porous medium. To summarize, the method proposed here can certainly be envisaged as a promising one to identify the hydrophilic pore fraction in 3D. It should be noted that the analysis was carried out using a single pair of relative permeability and capillary-pressure curves that were supposed to be obtained under quasistatic conditions, i.e., for an exceedingly small capillary number. Some additional data, obtained, for instance, with a small but finite capillary number, which would require taking viscous effects into account in the model, could also be considered to better constrain the inverse procedure and improve the estimation of $f$.

Another concern lies in the wettability distribution under consideration both in terms of spatial scale and of local contact angle distribution. In the present study, a Boolean distribution was considered, corresponding to only two values of the contact angle. Also, as illustrated in Fig. 2 (see Refs. $[6,8]$ as well), the change in the contact angle has a significant impact on the capillary equilibrium pattern when one value of the contact angle corresponds to hydrophilicity and the other to hydrophobicity. The capillary-pressure and relative permeability curves sensitivity to $f$ is expected to be less when both contact angle values correspond to either hydrophilicity [for instance $\theta=\left(20^{\circ}, 60^{\circ}\right)$ ] or hydrophobicity [for example $\left.\theta=\left(115^{\circ}, 150^{\circ}\right)\right]$. In this respect, the method is likely to be more efficient when the change in the wettability is around the wettability transition marking a significant change in the displacement pattern [43], as considered in the present study. In addition, it would be interesting to explore the capabilities of the method for more complex situations than the one considered in the present article. For example, in direct continuation with this work, it would be of interest to consider the case where the local contact angle value is distributed over a given range, for instance between $80^{\circ}$ and $115^{\circ}$. The latter case could correspond to a gradual change in the wettability rather than an abrupt change from $115^{\circ}$ to $80^{\circ}$. Also, it has been reported that in practical situations of a GDL for instance, the spatial distribution of the PTFE coating can be quite heterogeneous but different from purely random, with PTFE present in the regions of the porous medium adjacent to its surface and nearly no PTFE deeper in the medium [44]. In other words, strong spatial correlations can exist in the local contact angle distributions and they are 
expected to have an impact on the relative and capillary-pressure curves. Therefore, it would be interesting to test the optimization method for this kind of situation as well.

A somewhat crude simplification sustaining the current approach was to consider a spatially uniform contact angle at the scale of each network element. In many situations, the local contact angle can vary within a pore body or throat. In this case, the characterization method considered in the present work could be used to determine the apparent contact angle associated with each network element. This apparent contact angle would reflect the contact angle spatial variations at the scale of the element. A much more ambitious approach would be to extent the method considering direct numerical simulations [32,33] instead of PNM simulations for computing the two-phase displacements. However, this would be dramatically more demanding in terms of computational resources. Furthermore, computation of quasistatic flows, i.e., two-phase flows at very low capillary numbers, with this type of method, is still an active research area [45,46].

As a final remark, it should be pointed out that the validation of the method on real porous media still represents a challenge. This stems from the fact that the local contact angle distribution cannot be obtained from commonly used experimental methods. An option could be to determine the contact angle distribution from the visualization of the fluid distribution and of the menisci inside the pore space [47]. Nevertheless, regardless of the difficulty for characterizing the local contact angle from the menisci shapes [22], it may also be difficult to obtain the contact angle distribution over a large enough region of the pore space with this method. A simpler validation test would be to use microfluidic devices of controlled patterned wettability [48].

\section{CONCLUSION}

In this work, a method dedicated to the identification of the wettability distribution within the pore space of a porous medium was proposed. The procedure combines two-phase flow pore network direct simulations and genetic and hill-climbing algorithms for the identification of the wettability properties treated as an inverse optimization problem. The minimization procedure relies on a fitness function involving the relative permeability of the displacing phase and the capillarypressure curves. The method was validated on a model problem where the network elements feature a Boolean contact angle, i.e., are either hydrophilic or hydrophobic. Results show that the fraction of the hydrophilic pore bodies and throats is properly identified testifying to the relevance of the approach to characterize the macroscopic wettability behavior of the medium and to provide a representative local contact angle map. Tests were successfully carried out on several media, which wettability properties are ranging from fully hydrophilic to fully hydrophobic. The very promising capabilities of the method opens wide perspectives for the characterization of wettability properties in more complex situations.

\section{ACKNOWLEDGMENTS}

This research was supported by the Project "PEMFC-SUDOE"-SOE1/P1/E0293, which is cofinanced by the European Regional Development Fund in the framework of the Interreg Sudoe programme.

[1] S. Park, J.-W. Lee, and B. N. Popov, A review of gas diffusion layer in PEM fuel cells: Materials and designs, Int. J. Hydrogen Energy 37, 5850 (2012).

[2] F. Barbir, PEM Fuel Cells: Theory and Practice (Academic Press, Amsterdam, 2012).

[3] N. R. Morrow, Wettability and its effect on oil recovery, J. Pet, Technol. 42, 1476 (1990).

[4] M. Lazghab, K. Saleh, I. Pezron, P. Guigon, and L. Komunjer, Wettability assessment of finely divided solids, Powder Technol. 157, 79 (2005). 
[5] F. J. Navarro-Brull and R. Gómez, Modeling pore-scale two-phase flow: How to avoid gas-channeling phenomena in micropacked-bed reactors via catalyst wettability modification, Ind. Eng. Chem. Res. 57, 84 (2018).

[6] S. P. Kuttanikkad, M. Prat, and J. Pauchet, Pore-network simulations of two-phase flow in a thin porous layer of mixed wettability: Application to water transport in gas diffusion layers of proton exchange membrane fuel cells, J. Power Sources 196, 1145 (2011).

[7] J. Pauchet, M. Prat, P. Schott, and S. P. Kuttanikkad, Performance loss of proton exchange membrane fuel cell due to hydrophobicity loss in gas diffusion layer: Analysis by multiscale approach combining pore network and performance modelling, Int. J. Hydrogen Energy 37, 1628 (2012).

[8] L. Ceballos and M. Prat, Slow invasion of a fluid from multiple inlet sources in a thin porous layer: Influence of trapping and wettability, Phys. Rev. E 87, 043005 (2013).

[9] O. Chapuis, M. Prat, M. Quintard, E. Chane-Kane, O. Guillot, and N. Mayer, Two-phase flow and evaporation in model fibrous media: Application to the gas diffusion layer of PEM fuel cells, J. Power Sources 178, 258 (2008).

[10] E. Amott, Observations relating to the wettability of porous rock, Pet. Trans., AIME 216, 156 (1959).

[11] E. C. Donaldson, R. D. Thomas, and P. B. Lorenz, Wettability determination and its effect on recovery efficiency, Soc. Pet. Eng. J. 9, 13 (1969).

[12] A. B. Dixit, J. S. Buckley, S. R. McDougall, and K. S. Sorbie, Empirical measures of wettability in porous media and the relationship between them derived from pore-scale modelling, Transp. Porous Media 40, 27 (2000).

[13] M. Fleury and F. Deflandre, Quantitative evaluation of porous media wettability using NMR relaxometry, Magn. Reson. Imag. 21, 385 (2003).

[14] D. N. Staicopolus, The computation of surface tension and of contact angle by the sessile-drop method, J. Colloid Sci. 17, 439 (1962).

[15] E. W. Washburn, The dynamics of capillary flow, Phys. Rev. 17, 273 (1921).

[16] V. Gurau, M. J. Bluemle, E. S. De Castro, Y. M. Tsou, J. A. Mann, Jr., and T. A. Zawodzinski, Jr., Characterization of transport properties in gas diffusion layers for proton exchange membrane fuel cells: 1. Wettability (internal contact angle to water and surface energy of GDL fibers), J. Power Sources 160, 1156 (2006).

[17] V. Parry, E. Appert, and J. C. Joud, Characterisation of wettability in gas diffusion layer in proton exchange membrane fuel cells, Appl. Surf. Sci. 256, 2474 (2010).

[18] D. K. Owens and R. C. Wendt, Estimation of the surface free energy of polymers, J. Appl. Polym. Sci. 13, 1741 (1969).

[19] M. Khishvand, A. H. Alizadeh, and M. Piri, In-situ characterization of wettability and pore-scale displacements during two- and three-phase flow in natural porous media, Adv. Water Resour. 97, 279 (2016).

[20] M. J. Blunt, T. Akai, and B. Bijeljic, Evaluation of methods using topology and integral geometry to assess wettability, J. Colloid Interface Sci. 576, 99 (2020).

[21] R. T. Armstrong, C. Sun, P. Mostaghimi, S. Berg, M. Rücker, P. Luckham, A. Giorgiadis, and J. E. McClure, Transport in Porous Media (2021), doi: 10.1007/s11242-021-01615-0.

[22] V. Cnudde and M. N. Boone, High-resolution X-ray computed tomography in geosciences: A review of the current technology and applications, Earth Sci. Rev. 123, 1 (2013).

[23] T. Agaesse, A. Lamibrac, F. Buechi, J. Pauchet, and M. Prat, Validation of pore network simulations of ex-situ water distributions in a gas diffusion layer of proton exchange membrane fuel cells with X-ray tomographic images, J. Power Sources 331, 462 (2016).

[24] J. Joos, T. Carraro, A. Weber, and E. Ivers-Tiffée, Reconstruction of porous electrodes by FIB/SEM for detailed microstructure modeling, J. Power Sources 196, 7302 (2011).

[25] D. J. Fairweather, P. Cheung, and D. T. Schwartz, The effects of wetproofing on the capillary properties of proton exchange membrane fuel cell gas diffusion layers, J. Power Sources 195, 787 (2010).

[26] S. Skiena, The Algorithm Design Manual, 2nd ed. (Springer, New York, 2010).

[27] E. Unsal, J. H. Dane, and G. V. Dozier, A genetic algorithm for predicting pore geometry based on air permeability measurements, Vadose Zone J. 4, 389 (2005). 
[28] S. C. Su, C. J. Lin, and C. K. Ting, An effective hybrid of hill climbing and genetic algorithm for 2D triangular protein structure prediction, Proteome Sci. 9, S19 (2011).

[29] O. Maalal, M. Prat, R. Peinador, and D. Lasseux, Determination of the throat size distribution of a porous medium as an inverse optimization problem combining pore network modeling and genetic and hill climbing algorithms, Phys. Rev. E 103, 023303 (2021).

[30] M. J. Blunt, M. D. Jackson, M. Piri, and P. H. Valvatne, Detailed physics, predictive capabilities and macroscopic consequences for pore-network models of multiphase flow, Adv. Water Resour. 25, 1069 (2002).

[31] M. J. Blunt, Flow in porous media-pore-network models and multiphase flow, Curr. Opin. Colloid Interface Sci. 6, 197 (2001).

[32] Z. Niu, Y. Wang, K. Jiao, and Z. J. Wu, Two-phase flow dynamics in the gas diffusion layer of proton exchange membrane fuel cells: Volume of fluid modeling and comparison with experiment, J. Electrochem. Soc. 165, F613 (2018).

[33] W. Chen and F. Jiang, Impact of PTFE content and distribution on liquid-gas flow in PEMFC carbon paper gas distribution layer: 3D lattice Boltzmann simulations, Int. J. Hydrogen Energy 41, 8550 (2016).

[34] J. T. Gostick, Versatile and efficient pore network extraction method using marker-based watershed segmentation, Phys. Rev. E 96, 023307 (2017).

[35] A. Q. Raeini, B. Bijeljic, and M. J. Blunt, Generalized network modeling: Network extraction as a coarsescale discretization of the void space of porous media, Phys. Rev. E 96, 013312 (2017).

[36] L. Giorgi, E. Antolini, A. Pozio, and E. Passalacqua, Influence of the PTFE content in the diffusion layer of low-Pt loading electrodes for polymer electrolyte fuel cells, Electrochim. Acta 43, 3675 (1998).

[37] R. Lenormand and C. Zarcone, Role of roughness and edges during imbibition in square capillaries, in: SPE Annual Technical Conference and Exhibition (1984).

[38] V. Mani and K. Mohanty, Effect of pore-space spatial correlations on two-phase flow in porous media, J. Pet. Sci. Eng. 23, 173 (1999).

[39] M. Rebai and M. Prat, Scale effect and two-phase flow in a thin hydrophobic porous layer. Application to water transport in gas diffusion layers of PEM fuel cells, J. Power Sources 192, 534 (2009).

[40] V. Joekar-Niasar, S. M. Hassanizadeh, and A. Leijnse, Insights into the relationships among capillary pressure, saturation, interfacial area and relative permeability using pore-network modelling, Transp. Porous Media 74, 201 (2008).

[41] K. Mecke, Integral geometry and statistical physics, Int. J. Mod. Phys. B 12, 861 (1998).

[42] D. Stauffer and A. Aharony, Introduction to Percolation Theory (Taylor \& Francis, London, 1992).

[43] M. Cieplak and M. O. Robbins, Dynamical Transition in Quasistatic Fluid Invasion in Porous Media, Phys. Rev. Lett. 60, 2042 (1988).

[44] H. Ito, K. Abe, M. Ishida, A. Nakano, T. Maeda, T. Munakata, H. Nakajima, and T. Kitahara, Effect of through-plane distribution of polytetrafluoroethylene in carbon paper on in-plane gas permeability, J. Power Sources 248, 822 (2014).

[45] M. Shams, A. Q. Raeini, M. J. Blunt, and B. Bijeljic, A numerical model of two-phase flow at the microscale using the volume-of-fluid method, J. Comput. Phys. 357, 159 (2018).

[46] H. Si Hadj Mohand, H. Hoang, G. Galliero, and D. Legendre, On the use of a friction model in a Volume of Fluid solver for the simulation of dynamic contact lines, J. Comput. Phys. 393, 29 (2019).

[47] M. G. Andrew, B. Bijeljic, and M. J. Blunt, Pore-scale contact angle measurements at reservoir conditions using x-ray microtomography, Adv. Water Resour. 68, 24 (2014).

[48] K. Ma, J. Rivera, G. J. Hirasaki, and S. L. Biswal, Wettability control and patterning of PDMS using UV-ozone and water immersion, J. Colloid Interface Sci. 363, 371 (2011). 